\title{
Adherence to self-care behaviours and associated barriers in type 2 diabetes patients of low-and middle-income countries: a systematic review protocol
}

Victor Mogre ${ }^{1,2^{*}}$ (D), Natalie A. Johnson ${ }^{1,3}$, Flora Tzelepis ${ }^{1,3,4}$, Jonathan Shaw ${ }^{5}$ and Christine Paul ${ }^{1,3}$

\begin{abstract}
Background: Diabetes has become a global health emergency affecting high-, middle- and low-income countries. Previous systematic reviews have either focused on patients' adherence to diabetes self-care behaviours only or barriers to diabetes care (including self-care) only in the published literature and have not also analysed data separately for low- and middle-income countries (LMICS). Thus, none have focused on adherence with, and barriers to, self-care behaviours from the perspectives of both patient and providers in low- and middle-income countries (LMICs). This systematic review will evaluate the published literature on adherence to five diabetes self-care behaviours (i.e., diet, exercise, self-monitoring of blood glucose, medication taking and foot care) and associated barriers in type 2 diabetes patients in LMICs. Healthcare providers' barriers to the provision of diabetes self-care support will also be reviewed.
\end{abstract}

Methods: This narrative review will be reported in accordance with the guidelines of the Preferred Reporting Items for Systematic review and Meta-Analysis Protocols (PRISMA-P). The electronic databases, MEDLINE, EMBASE, CINAHL, SCOPUS, PsycINFO, Cochrane Library and the British Nursing Index will be searched. Qualitative and quantitative studies reporting on type 2 diabetes patients' adherence to self-care behaviours and associated barriers in LMICs will be included. Studies also reporting on barriers encountered by providers in LMICs providing diabetes care and supporting patients to adhere to self-care behaviours will also be included. Cross-sectional studies, observational cohort studies, baseline data of randomised controlled trials and qualitative studies will be eligible. Two independent reviewers will screen articles for inclusion, undertake quality assessment of included studies and execute data extraction using standardised forms. Discrepancies will be discussed to reach consensus, and another reviewer will adjudicate if the need arises. The Guidance of Narrative Synthesis in Systematic Reviews will be employed to explore relationships within and between included studies.

Discussion: This review will provide evidence on adherence to self-care behaviours by type 2 diabetes patients in LMICs. Barriers experienced by patients in LMICs to adhere to recommended self-care behaviours will also be identified. Barriers experienced by healthcare providers in LMICs in providing self-care support patients will also be determined.

\section{Systematic review registration: PROSPERO CRD42016035406}

Keywords: Adherence, Barriers, Self-care, Type 2 diabetes, Low- and middle-income countries

\footnotetext{
* Correspondence: vmogre@uds.edu.gh

'School of Medicine and Public Health, University of Newcastle, University

Drive, Callaghan, New South Wales 2308, Australia

2Department of Health Professions Education, School of Medicine and Health

Sciences, University for Development Studies, P. O. Box TL 1883, Tamale,

Ghana

Full list of author information is available at the end of the article
} 


\section{Background}

Diabetes affected 422 million adults in 2014 compared to 108 million in 1980 [1]. The increasing prevalence is associated with an increase in the prevalence of risk factors such as excess body weight, sedentary lifestyle, diet and ageing populations [2, 3]. In the last 10 years, the prevalence of diabetes has risen faster in low- and middleincome countries than in high-income countries [1]. The World Health Organization (WHO) anticipates that infectious diseases, maternal and infant mortality and malnutrition will be overtaken by non-communicable diseases such as diabetes mellitus (DM) in low- and middleincome countries (LMICs) by 2030 [4, 5]. Characterised by hyperglycaemia, insulin resistance and relative insulin deficiency, type $2 \mathrm{DM}$ is the most frequently occurring form of DM globally [6-8].

Diabetes patients of LMICs are at increased risk of late diagnosis, poor diabetes control, hospitalisations and the development of diabetes-related complications [9, 10]. Also a higher proportion of premature deaths due to high blood glucose occurred in LMICs than in highincome countries [1].

Being a complex chronic disorder, diabetes care requires regular attention to diet, physical activity, monitoring of blood sugar, medications and foot care to attain positive health outcomes [11]. As has been advocated by clinical practice guidelines from the USA, UK and globally [12-15], these activities are referred to as diabetes self-care behaviours requiring the active participation of the patient in his or her care [15]. As each of these behaviours is important to health outcomes, it is essential to have a clear understanding of the level of adherence to each self-care behaviour and barriers to the performance of each of them for LMICs.

A number of reviews have examined adherence to selfcare behaviours among type 2 diabetes patients [15] and barriers to diabetes care [16-18] including self-care [19]. The review by Coyle et al. included studies from lowand middle-income countries (LMICs) but only evaluated patients' adherence to self-care behaviours (i.e. did not report on patients' barriers to self-care) and did not analyse data from LMICs separately. The reviews by Pun et al. and Nam et al. [16-18] also included studies from LMICs, examined patient and provider barriers to general diabetes care including self-care but did not consider barriers to self-care behaviours separately and did not analyse LMICs data separately. Also, they did not undertake quality assessment of the included studies. The review by Sohal et al. evaluated patient and provider barriers and facilitators to diabetes management using only studies conducted in South Asia [18]. Wilkinson et al. [19] reviewed the literature to identify factors that influence patients' ability to self-care for their diabetes, but only included qualitative studies, considered both type 1 and type 2 diabetes patients, did not include studies among providers and did not analyse LMICs data separately. Accordingly, a systematic review of the published literature from LMICs on patient adherence with, and the barriers to, self-care behaviours from the perspectives of both the patient and providers is warranted.

This systematic review will examine both qualitative and quantitative studies conducted in LMICs related to:

a. Type 2 diabetes patients' adherence to five self-care behaviours: recommended diet, sufficient exercise, diabetes medications, self-monitoring of blood glucose and foot care.

b. Barriers to self-care behaviours among patients with type 2 diabetes

c. Barriers to the provision of appropriate care to patients with type 2 diabetes among healthcare providers.

\section{Methods}

This systematic review will be reported in accordance with the guidelines of the Preferred Reporting Items for Systematic review and Meta-Analysis Protocols (PRISMA-P) 2015 [20]. Details of this can be found in an additional file (see Additional file 1).

\section{Definition of low- and middle-income countries}

Based on the 2015 Gross National Income per capita (GNI), low-income countries are those with a GNI per capita of $\leq \mathrm{US} \$ 1,045$ and middle-income countries are those with a GNI per capita of >US\$1,045 but $<$ US\$12,736 [21]. Any country listed by World Bank as a LMIC at any point during the review period will be included.

\section{Information sources}

Electronic databases including MEDLINE (OVID interface, 1948 and beyond), EMBASE (OVID interface, 1980 onwards), CINAHL, SCOPUS, PsycINFO, Cochrane Library and the British Nursing Index will be searched. The websites of relevant organisations such as the International Diabetes Federation (IDF), WHO and other diabetes-related institutions will also be examined for other potentially relevant studies. To ensure relevant publications are captured, the reference lists of relevant studies or systematic reviews will be hand searched.

\section{Search strategy}

The databases will be searched using medical subject headings $(\mathrm{MeSH})$ and free text words relating to the themes of this review (Table 1). Truncation and appropriate Boolean operators will be incorporated into the search strategy to cater for the different use of terms. Limitations pertaining to the inclusion and exclusion 
Table 1 Search strategy

\begin{tabular}{|c|c|}
\hline \multicolumn{2}{|c|}{ Searches } \\
\hline 1 & diabetes mellitus.mp. or Diabetes Mellitus/ \\
\hline 2 & $\begin{array}{l}\text { Diabetes Mellitus, Type 2/or Diabetes Mellitus, or diabetes } \\
\text { mellitus type 2.mp. }\end{array}$ \\
\hline 3 & $\begin{array}{l}\text { (non insulin*dep* or noninsulin*dep*).mp. [mp }=\text { title, } \\
\text { abstract, original title, name of substance word, subject } \\
\text { heading word, keyword heading word, protocol } \\
\text { supplementary concept word, rare disease supplementary } \\
\text { concept word, unique identifier] }\end{array}$ \\
\hline 4 & 1 or 2 or 3 \\
\hline 5 & self-care.mp. or Self Care/ \\
\hline 6 & self-manage ${ }^{*} . m p$. or Patient Education as Topic/ \\
\hline 7 & self-care behaviours.mp. \\
\hline 8 & 5 or 6 or 7 \\
\hline 9 & diet.mp. or Diet/ \\
\hline 10 & Food Habits/or dietary habit*.mp. \\
\hline 11 & nutrition.mp. \\
\hline 12 & 9 or 10 or 11 \\
\hline 13 & exercise.mp. or Exercise/ \\
\hline 14 & physical activity.mp. \\
\hline 15 & 13 or 14 \\
\hline 16 & Blood Glucose Self-Monitoring/ \\
\hline 17 & Self Care/or blood glucose testing.mp. \\
\hline 18 & 16 or 17 \\
\hline 19 & Self Care/or foot care.mp. \\
\hline 20 & $\begin{array}{l}\text { Patient Compliance/or medication taking.mp. or Self } \\
\text { Administration/or Medication Adherence/ }\end{array}$ \\
\hline 21 & adherence.mp. \\
\hline 22 & 20 or 21 \\
\hline 23 & barrier.mp. \\
\hline 24 & $\begin{array}{l}\text { Health Services Accessibility/or Health Personnel/or patient } \\
\text { barrier.mp. or "Attitude of Health Personnel"/ }\end{array}$ \\
\hline 25 & 23 or 24 \\
\hline 26 & $\begin{array}{l}\text { Developing Countries/or Socioeconomic Factors/or Low- } \\
\text { income countries.mp. }\end{array}$ \\
\hline 27 & Developing Countries/or middle-income countries.mp. \\
\hline 28 & 26 or 27 \\
\hline 29 & 4 or 8 or 12 or 15 or 18 or 19 or 22 or 28 \\
\hline 30 & 25 and 29 \\
\hline 31 & $\begin{array}{l}\text { limit to (english language and yr = "1990 -Current" and "all } \\
\text { adult ( } 19 \text { plus years)") }\end{array}$ \\
\hline
\end{tabular}

criteria will be set. An Ovid MEDLINE strategy will be developed by VM and other members of the research team under the guidance of a librarian with experience in systematic review searching. The finalized Ovid MEDLINE strategy will be adapted to the syntax and subject headings of the other databases. The search strategy will be reviewed by another librarian in accordance with the
Peer Review of Electronic Search Strategies (PRESS) checklist. The execution of the search strategy will be done by VM.

\section{Eligibility criteria}

Studies will be included in accordance with the criteria outlined below.

a. Type of data: Studies using quantitative, qualitative or both approach(es) will be included. Included studies will be limited to published papers including published theses/dissertations.

b. Study design: Cross-sectional studies, cohort studies, baseline data from randomised controlled trials (RCTs) and qualitative studies (e.g. in depth interviews, focus groups) will be eligible for inclusion.

c. Study participants: (i) Adults with type 2 diabetes from LMICs. Studies where the majority ( $\geq 50 \%$ ) of participants are from a LMIC will be included. (ii) Studies that report on the barriers faced by healthcare professionals who deliver care to type 2 diabetes patients in LMICs will also be eligible for inclusion.

d. Search limitations: All studies published from January 1990 onwards will be included. This time frame is informed by global estimates of diabetes that recognised diabetes as a global disease affecting adult populations of developing countries or LMICs [22].

e. Study focus: Studies that are concerned with patient adherence to self-care behaviours and barriers to self-care. Also, studies that report on barriers experienced by providers to support patients to self-care for their diabetes will be included.

f. Setting: Both population- and clinic-based studies will be eligible for inclusion.

g. Language: Due to funding constraints, only studies published in English will be eligible for inclusion

\section{Exclusion criteria}

Studies will be excluded if the majority of participants were younger than 18 years or had gestational diabetes. Conference proceedings, non-peer reviewed papers, opinion pieces, commentaries, case reports, abstracts and systematic reviews will be excluded.

\section{Data management, screening and selection}

All search results will be downloaded into the reference manager ENDNOTE version X7 for screening. The liberal accelerated screening method for the screening of the literature search results will be adopted [23]. Firstly, VM will screen the title, abstract and subject headings of the citations in accordance with the eligibility criteria. Those that meet the eligibility criteria will be included and moved into the next level of assessment as well as 
those that were unclear. Those that are excluded as per the eligibility criteria will be reviewed by another member of the review team to confirm exclusion or otherwise. Secondly, full-text screening will be carried out by two members of the review team independently. Discussions and consensus building between the two reviewers will be used to resolve discordance and one or two arbitrators will adjudicate unresolved disagreements. In accordance with the PRISMA flow diagram, the entire selection process and reasons for exclusion will be recorded [24, 25]. Questions for screening will be developed in accordance with the eligibility criteria for the two-stage assessment of potentially eligible studies. The screening questions will be piloted, prior to the formal screening processes.

\section{Characteristics of included studies}

The content of each included study will be extracted by two independent reviewers and recorded on a standard data collection form utilised in other reviews [24]. The following data will be extracted:

a. Publication details: author(s) names, year of publication, country of study and years data were collected

b. Study setting: population based, clinic-based

c. Study design: cross-sectional, cohort, baseline data from RCT, qualitative (focus groups, in-depth interviews)

d. Details of study population: demographics, sample size, eligibility criteria

e. Sampling methods: consent rates

f. Measures

g. Results

During the data extraction process, disagreements will be resolved through discussion and consensus building and one or two arbitrators will adjudicate if needed.

\section{Quality assessment}

All quantitative studies will be assessed using the National Heart, Lung and Blood Institute (NHLBI) standardised quality rating tools for quality assessment [26]. The NHLBI standardised tools are study-design specific $[26,27]$. Studies having observational cohort and crosssectional designs will be assessed using the NHLBI standardized Quality Assessment Tool for Observational Cohort and Cross-sectional studies. Data only from the baseline phase of RCTs will be included in this review because adherence to self-care behaviours and reporting of barriers may change at follow-up as a result of an intervention. Baseline data only from an RCT represents a cross-sectional assessment and therefore the NHLBI standardized Quality Assessment Tool for Observational
Cohort and Cross-sectional studies will also be used for such studies.

The NLBI tools will allow for assessment of potential flaws in study methods including sources of bias, sampling, confounding, study power and other relevant factors. Each study will be judged as "good", "fair" or "poor" quality based on ratings of a list of items included in the tools. Globally, a good study has the least risk of bias and is considered valid. A fair study is prone to some bias but insufficient to invalidate its findings, varying in its strengths and weaknesses. A poor study has high risk of bias and is considered invalid. Quality assessment will be conducted independently by two members of the review team. Differences will be resolved through discussions between these two and if unresolved the other members of the team will be consulted for adjudication.

Qualitative studies will be assessed according to quality assessment categories adopted by Popay, Rogers and Williams [28]. These categories will pertain to relevance and appropriateness of research design, study context, use of convenience/purposive sampling, richness of data, thoroughness of data analysis and clarity of interpretation and logical generalisation of findings based on theoretical underpinnings. This tool has been used widely for assessing the quality of qualitative studies in several systematic reviews [29-31]. It has been reported to be applicable across a wide range of qualitative methods [30, 32].

\section{Data analysis and synthesis}

A systematic narrative synthesis will be conducted. Tables and narrative summaries will be used to present the characteristics and findings of included studies. Relationships within and between included studies will be explored as well as assessment of the robustness of the synthesis, in accordance with the Guidance of Narrative Synthesis in Systematic reviews produced by the Economic and Social Research Council (ESRC) methods programme [33]. This guidance provides a framework for the execution of narrative synthesis using both broad and specific techniques and tools. It has been used widely in several systematic reviews [34-38]. VM will conduct the synthesis in consultation with the rest of the review team members.

\section{Discussion}

Systematically reviewing the published literature from LMICs will increase knowledge on the self-care behaviours that are commonly adhered to and those that are not among type 2 diabetes patients in LMICs. It will also provide data relating to barriers faced by patients to selfcare for their diabetes and providers to support patients to self-care in LMICs which will assist with the design of interventions to improve care planning and delivery in this population. The inclusion of both qualitative and 
quantitative studies will allow for a comprehensive review of the published literature.

\section{Additional files}

Additional file 1: PRISMA-P checklist. This checklist provides a list of recommended items to include in a systematic review protocol and the pages upon which those items are covered in the protocol. (DOCX $30 \mathrm{~kb}$ )

\section{Abbreviations}

ESRC: Economic and social research council; GNI: Gross national income pe capital; IDF: International diabetes federation; LMICs: Low- and middleincome countries; NHLBI: National Heart, Lung and Blood Institute; PRESS: Peer review of electronic search strategies; PRISMA-P: Preferred Reporting Items for Systematic Review and Meta-Analysis Protocol; RCTs: Randomised controlled trials; WHO: World Health Organization

\section{Acknowledgements}

The authors wish to thank Debbie Booth, a Senior Research Librarian at the University of Newcastle for her assistance in identifying the key words of the review. The authors also wish to acknowledge infrastructure support from the Hunter Medical Research Institute, Australia.

\section{Funding}

VM was supported by the Research Training Scheme of the University of Newcastle, Australia. CP was supported by an NHMRC career development fellowship.

\section{Availability of data and materials}

Not applicable.

\section{Authors' contributions}

VM conceived and designed the study, and drafting of manuscript; FT, NAJ, CP and JS jointly undertook critical revision of the manuscript. All authors approved the manuscript for publication.

\section{Competing interests}

The authors declare that they have no competing interests.

\section{Consent for publication}

Not applicable.

\section{Ethics approval and consent to participate}

Not applicable.

\section{Author details}

'School of Medicine and Public Health, University of Newcastle, University Drive, Callaghan, New South Wales 2308, Australia. ${ }^{2}$ Department of Health Professions Education, School of Medicine and Health Sciences, University for Development Studies, P. O. Box TL 1883, Tamale, Ghana. ${ }^{3}$ Hunter Medical Research Institute, Locked bag 1000, New Lambton, New South Wales 2305 Australia. ${ }^{4}$ Hunter New England Population Health, Hunter New England Local Health District, Locked Mail Bag 10, Wallsend, New South Wales 2287, Australia. ${ }^{5}$ Baker IDI Heart and Diabetes Institute, Melbourne, Victoria 3004 Australia.

Received: 6 December 2016 Accepted: 17 February 2017 Published online: 27 February 2017

\section{References}

1. WHO Global report on diabetes. Geneva: WHO; 2016.

2. International Diabetes Federation. IDF diabetes atlas. Brussels: International Diabetes Federation; 2013

3. Wild S, Roglic G, Green A, Sicree R, King H. Global prevalence of diabetes estimates for the year 2000 and projections for 2030. Diabetes Care. 2004 27:1047-53

4. World Health Organization. Global status report on noncommunicable diseases 2010. Geneva: World Health Organization; 2011.
5. Levitt NS. Diabetes in Africa: epidemiology, management and healthcare challenges. Heart. 2008:94:1376-82.

6. International diabetes federation. IDF Diabetes Atlas 7th Edition. Brussels, Belgium: IDF; 2015.

7. American Diabetes Association. Diagnosis and classification of diabetes mellitus. Diabetes Care. 2005;28:S37.

8. WHO. Definition, diagnosis and classification of diabetes mellitus and its complications: report of a WHO Consultation Part 1: diagnosis and classification of diabetes mellitus. 1999

9. Booth GL, Hux JE. Relationship between avoidable hospitalizations for diabetes mellitus and income level. Arch Intern Med. 2003:163:101-6.

10. Bazargan M, Johnson KH, Stein JA. Emergency department utilization among Hispanic and African-American under-served patients with type 2 diabetes. Ethn Dis. 2003;13:369-75.

11. Cramer JA. A systematic review of adherence with medications for diabetes. Diabetes Care. 2004;27:1218-24.

12. International Diabetes Federation Guideline Development Group. Global guideline for type 2 diabetes. Diabetes Res Clin Pract. 2014;104:1.

13. American Diabetes Association. Standards of medical care in diabetes_2014. Diabetes Care. 2014;37:S14-80.

14. National Collaborating Centre for Chronic Conditions (UK). Type 2 diabetes: national clinical guideline for management in primary and secondary care (update). UK: Royal College of Physicians; 2008.

15. Coyle ME, Francis K, Chapman Y. Self-management activities in diabetes care: a systematic review. Aust Health Rev. 2013;37:513-22.

16. Pun SP, Coates V, Benzie IF. Barriers to the self-care of type 2 diabetes from both patients' and providers' perspectives: literature review. J Nurs Healthc Chronic IIIn. 2009:1(1):4-19.

17. Nam S, Chesla C, Stotts NA, Kroon L, Janson SL. Barriers to diabetes management patient and provider factors. Diabetes Res Clin Pract. 2011:93(1):1-9.

18. Sohal T, Sohal P, King-Shier KM, Khan NA. Barriers and facilitators for type-2 diabetes management in South Asians: a systematic review. PLoS One. 2015;10:e0136202

19. Wilkinson A, Whitehead L, Ritchie L. Factors influencing the ability to selfmanage diabetes for adults living with type 1 or 2 diabetes. Int J Nurs Stud. 2014:51(1):111-22

20. Shamseer L, Moher D, Clarke M, Ghersi D, Liberati A, Petticrew M, Shekelle P, Stewart LA. Preferred reporting items for systematic review and meta-analysis protocols (PRISMA-P) 2015: elaboration and explanation. BMJ. 2015:349:97647.

21. World Bank. New country classifications. 2015. http://blogs.worldbank.org/ opendata/new-country-classifications.

22. King H, Rewers M. Global estimates for prevalence of diabetes mellitus and impaired glucose tolerance in adults. Diabetes Care. 1993;16:157-77.

23. Khangura S, Konnyu K, Cushman R, Grimshaw J, Moher D. Evidence summaries: the evolution of a rapid review approach. Syst Rev. 2012;1:1.

24. Young MM, Stevens A, Porath-Waller A, Pirie T, Garritty C, Skidmore B, Turner L, Arratoon C, Haley N, Leslie K. Effectiveness of brief interventions as part of the screening, brief intervention and referral to treatment (SBIRT) model for reducing the non-medical use of psychoactive substances: a systematic review protocol. Syst Rev. 2012;1:22.

25. Moher D, Liberati A, Tetzlaff J, Altman DG. Preferred reporting items for systematic reviews and meta-analyses: the PRISMA statement. Ann Intern Med. 2009;151:264-9.

26. Jorgensen CM. Measuring adherence to evidence-based practice guidelines for the management of hypertension in women. DNP Practice Inquiry Projects. 2015:Paper 28.

27. National Heart, Lung and Blood Institutue. Development and use of study quality assessment tools. 2014. Retrieved from http://www.nhlbi.nih.gov/ health-pro/guidelines/in-develop/cardiovascular-risk-reduction/tools/ background.

28. Popay J, Rogers A, Williams G. Rationale and standards for the systematic review of qualitative literature in health services research. Qual Health Res. 1998:8:341-51.

29. Attree P. Low-income mothers, nutrition and health: a systematic review of qualitative evidence. Matern Child Nutr. 2005:1:227-40.

30. Sim J, Madden S. Illness experience in fibromyalgia syndrome: a metasynthesis of qualitative studies. Soc Sci Med. 2008:67:57-67.

31. Yu DS, Lee DT, Kwong AN, Thompson DR, Woo J. Living with chronic heart failure: a review of qualitative studies of older people. J Adv Nurs. 2008;61: $474-83$. 
32. Zeh P, Sandhu HK, Cannaby AM, Sturt JA. Cultural barriers impeding ethnic minority groups from accessing effective diabetes care services: a systematic review of observational studies. Divers Equal Health Care. 2014;11:9-33.

33. Popay J, Roberts H, Sowden A, Petticrew M, Arai L, Rodgers M, Britten N, Roen K, Duffy S. Guidance on the conduct of narrative synthesis in systematic reviews. A product from the ESRC methods programme Version 2006, 1.

34. Whitehead PJ, Drummond A, Walker MF, Parry RH. Interventions to reduce dependency in personal activities of daily living in community dwelling adults who use homecare services: protocol for a systematic review. Syst Rev. 2013;2:49.

35. Rodgers M, Sowden A, Petticrew M, Arai L, Roberts H, Britten N, Popay J. Testing methodological guidance on the conduct of narrative synthesis in systematic reviews effectiveness of interventions to promote smoke alarm ownership and function. Evaluation. 2009;15:49-73.

36. Arai L, Britten N, Popay J, Roberts H, Petticrew M, Rodgers M, Sowden A. Testing methodological developments in the conduct of narrative synthesis: a demonstration review of research on the implementation of smoke alarm interventions. Evid Policy. 2007;3:361-83.

37. Centre for Reviews and Dissemnination. CRD's guidance for undertaking reviews in health care. York: Centre for Reviews and Dissemination; 2009

38. McDaid C, Trowman R, Golder S, Hawton K, Sowden A. Interventions for people bereaved through suicide: systematic review. Br J Psychiatry. 2008;193:438-43.

\section{Submit your next manuscript to BioMed Central and we will help you at every step:}

- We accept pre-submission inquiries

- Our selector tool helps you to find the most relevant journal

- We provide round the clock customer support

- Convenient online submission

- Thorough peer review

- Inclusion in PubMed and all major indexing services

- Maximum visibility for your research

Submit your manuscript at www.biomedcentral.com/submit

) Biomed Central 